\title{
Correction to: Accelerated quantification of tissue sodium concentration in skeletal muscle tissue: quantitative capability of dictionary learning compressed sensing
}

\author{
Matthias Utzschneider ${ }^{1,2} \mathbb{D} \cdot$ Nicolas G. R. Behl ${ }^{3} \cdot$ Sebastian Lachner $^{1} \cdot$ Lena V. Gast $^{1} \cdot$ Andreas Maier $^{2}$. \\ Michael Uder ${ }^{1} \cdot$ Armin M. Nagel ${ }^{1,3,4}$
}

Published online: 12 February 2020

(c) European Society for Magnetic Resonance in Medicine and Biology (ESMRMB) 2020

\section{Correction to: \\ Magnetic Resonance Materials in Physics, Biology and Medicine \\ https://doi.org/10.1007/s10334-019-00819-2}

The original version of this article unfortunately contained a mistake. Title was incorrect.

The corrected title is given below.

Accelerated quantification of tissue sodium concentration in skeletal muscle tissue: quantitative capability of dictionary learning compressed sensing.
The original article has been corrected.

Publisher's Note Springer Nature remains neutral with regard to jurisdictional claims in published maps and institutional affiliations.

The original article can be found online at https://doi.org/10.1007/ s10334-019-00819-2.

Matthias Utzschneider

matthias.utzschneider@uk-erlangen.de

1 Institute of Radiology, University Hospital Erlangen, Friedrich-Alexander-Universität Erlangen-Nürnberg (FAU), Maximiliansplatz 3, 91054 Erlangen, Germany

2 Pattern Recognition Lab, Department of Computer Science, Friedrich-Alexander-Universität Erlangen-Nürnberg (FAU), Erlangen, Germany

3 Division of Medical Physics in Radiology, German Cancer Research Center (DKFZ), Heidelberg, Germany

4 Institute of Medical Physics, Friedrich-Alexander-Universität Erlangen-Nürnberg (FAU), Erlangen, Germany 